\title{
METAMODELLING APPROACH AND SOFTWARE TOOLS FOR PHYSICAL MODELLING AND SIMULATION
}

\author{
VitaliyMezhuyev ${ }^{1}$, Felipe Pérez-Rodríguez ${ }^{2}$ \\ ${ }^{1}$ Department of Informatics and Software Engineering, \\ Berdyansk State Pedagogical University, Ukraine \\ ${ }^{2}$ Instituto de Física, Benemérita Universidad Autónoma de Puebla, Mexico \\ E-mail: mejuev@ukr.net
}

\begin{abstract}
In computer science, metamodelling approach becomes more and more popular for the purpose of software systems development. In this paper, we discuss applicability of the metamodelling approach for development of software tools for physical modelling and simulation.To define a metamodel for physical modelling the analysis of physical models will be done. The result of such the analyses will show the invariant physical structures, we propose to use as the basic abstractions of the physical metamodel. It is a system of geometrical objects, allowing to build a spatial structure of physical models and to set a distribution of physical properties. For such geometry of distributed physical properties, the different mathematical methods can be applied. To prove the proposed metamodelling approach, we consider the developed prototypes of software tools.
\end{abstract}

Keywords: Metamodelling;Physical Modelling and Simulation; Geometrical Metamodel;Software Tools;Metamaterials Design.

\section{INTRODUCTION}

The modelling tools play an important part on the market of scientific and engineering software. At the same time, the technology of modelling has quite different specifics in computer engineering and physical science: while in the first case, the process of modelling may be considered as building UML (uml-forum) diagrams, in the second case it may be implementation of solution by systems of differential equations. However, in both cases, a modelling is development of an ideal copy of an object by allocation of its significant properties with the help of the specially designed computer tools.

The question is what will be the next stage of development of the software tools for physical modelling and simulation? The rising complexity of physical systems inevitably results in the increasing level of abstraction of concepts, have used for the modelling.

Available mathematical software for physical modelling as e.g. (mathworks; mathsoft; maplesoft) allow us to apply a wide spectrum of methods, but are complex for the end user, due to modelling with highly abstract concepts. Existing domain-specific modelling systems can solve only a certain class of problems in the corresponding physical domains - mechanics, molecular physics, electrodynamics, optics, atomic and quantum physics.

For this reason, elaboration of the new principles for development of software tools for physical modelling and simulation is needed. In the paper, we propose to use the metamodelling approach, which allows covering a wide range of physical domains and at 
the same time can be applied by users, having no strong mathematical background. A formally defined physical metamodel allows us to produce multiple domain-specific models. As against other modelling approaches, which use textual modelling languages, we define geometrical objects for setting distribution of the physical data, and allow manipulations with geometrical objects as application of formal mathematical and programming operators.

The language of physics is mathematics; therefore, mathematical formalisms have to be used for physical models development. The idea of proposed approach is using two interrelated languages: the symbolical mathematical language (at the level of the metamodel development by the expert of a domain) and the language of geometrical structures (at the level of a model development by the end user of a software tool). In such a case, the mathematics is mostly hidden for the user, because an expert of domain does development of the metamodel.

Such an approach significantly facilitates the process of physical modelling (with using theoretical methods), research and understanding of solution (with using model experiments). As result, it significantly decreases the time between development of a physical model, its investigation and realisation of a solution.

To develop the metamodelling approach we should take into account:

1. The specifics of physical models (i.e. their significant properties, like existence in space in time; applicability of mathematical methods etc.).

2. The specifics of physical cognition (i.e. the technology of physical research both at theoretical and experimental levels).

Therefore, elaboration of the metamodelling approach needs investigation of properties and relationships of physical models. This allow us to derive invariant structures, needed for representation, development and investigation of physical models. It also shows mathematical methods, applicable for corresponding data structures in software tools.

In this paper, we will emphasize on the spatial structure of physical domains. The object of our research is the structures of physical models and their representation by other correlated systems (mathematical, geometrical and information). The goal of the paper is development of the new metamodelling approach as result of analysis of physical models, mathematical formalisms, geometrical and information structures.

The next section of the paper briefly introduces the existing metamodelling approaches for software systems development. Next, we consider physical models to derive invariant structures for physical metamodelling.

Section 3 consider formal semantics of proposed geometrical meta-metamodel. Section 4 discusses implementation of software prototypes. Discussion, further tasks and conclusion finalize the paper.

\section{BASIC IDEAS OF THE METAMODELLING APPROACH FOR SOFTWARE SYSTEMS DEVELOPMENT}

Along with the possibility of covering multiple domains, universal modelling languages, the most prominent of which is Unified Modelling Language (UML), have many drawbacks. The necessity of models development for a variety of domains leads to the situation, that modelling concrete aspects of specific domains remains outside of possibilities of the universal languages. In this case, to support the modelling specific 
properties of domains the dialects of universal languages are developed. The big list of UML profiles (Catalog of UML), in fact, fully negates the idea of its "universality".

The idea of Domain-Specific Modeling (DSM) is the developments of special languages that allow us effectively capture the domain properties for use in software systems design. Such the Domain-Specific Languages (DSLs) with the help of a certain meta-metamodel are developed. There are several meta-metamodels, for example, GOPPRR (Graph-Object-Property-Port-Role-Relationship) is used in the MetaEdit+ toolset (Steven and Tolvanen, 2008). There are multiple examples of using graphs for the metamodelling (see e.g., (Gregory, 1999)), which provide a natural way for the decomposition of domains into hierarchical structures. Emphasizing the power of these approaches, existing metamodels cannot effectively capture the properties of multidimensional physical domains. To contribute to this area, a new metamodeling approach for designing cyber-physical systems was proposed in the paper (Vitaliy and Refik, 2013).

The metamodelling architecture allows us adequately capture and represent the specific properties of multidimensional cyber-physical domains. With multidimensionality, we refer to a property of the domains in which the modelling must use abstractions that have different dimensions (1D, 2D, 3D and even $4 \mathrm{D}$, in cases of the expression of time semantics).

In the (Vitaliy, 2014; Vitaliy, 2015) we expanded the approach and proposed the methodology of Domain-Specific Mathematical Modelling (DSMM), allowing development of metamodels and their application to modelling domains with different mathematical structure.

The metamodel was defined as a formal system, which includes alphabet of types, grammar, and operations. The additional level of the metamodelling architecture was introduced, allowing developing meta-metamodels in different mathematical semantics. This approach improves DSM, where the mathematical structure of the meta-metamodels is hardcoded and development of new formalisms is impossible. The software architecture of the DSMM tools was considered in (Vitaliy, 2014).

\section{ANALYSIS OF PHYSICAL MODELS AND DEVELOPMENT OF GEOMETRICAL METAMODEL}

To cover a wide spectrum of physical models we need allocate the concepts, reflecting the most general principles of the physical science. These fundamental principles (to which the existence of physical domains in space in time concern) determine the ways of development and transformation of computer models.

First, it is necessary to understand the structure of physical models, and it is the spatial and the time structure. Physical domains exist in space and time, what can be recognized as the basic abstraction behind all the physical models.

The existence of a real object in space and time is reflected by its spatial and time parameters, describing an object in a physical law. The time has one dimension, i.e. one parameter can fix an event. The real space has three dimensions; that is three parameters can fix the location of any point of a physical object. These statements are the basic properties of a space and a time, to be used in the metamodel for fixing spatial and time attributes of the physical models (time interval, shape, size, spatial structure etc.).

Let's also briefly consider specifics of a physical cognition. Any part of physical reality can be an object of research. To determine an object of research means to find out 
its distinctive properties. In physics, the properties of an object are fixed by physical values (for example the mass $m$ shows the gravitational and inertial properties of bodies, the charge $q$-electromagnetic properties and so on). Such the attributes cause the possible forms of interaction (in the physical context - gravitational, electromagnetic, strong and weak) and so define physical behaviour.

Therefore, another important abstraction at developing physical models is the assumption about spatial distribution of physical properties. For example, a material point is the model of a physical object abstracted from its shape, size and structure. In other words, we consider the properties of a physical object as located at a geometrical point.

There are other well-known models and abstractions, which use the concept of spatial distribution of physical properties. E.g., the linear distribution of physical properties is used in the model of a thin conductor with a current; the model of the surface distribution of a charge; different kinds of spatial distributions of scalar and vector properties (for example, the scalar field of the potential of a system of charges or the vector field of the magnetic induction produced by a conductor with a current) are also well known in physics.

Thus, the abstraction concerning spatial distribution of physical properties is widely used when developing physical models from different domains. And the use of relevant mathematical formalisms results from the applicability of such the abstractions.

Solving the one-, the two- and the three-dimensional physical problems by the integro-differential calculus is based on modelling the relevant distribution of properties (linear, surface and volumetric), where integration and differentiation of the first, second and third orders are accordingly applied. Hence, definition of the meta-metamodel concepts, implementing such the basic spatial distributions of physical properties will facilitate the process of mathematical modelling. In general, the abstractions of the metametamodel should reflect the discrete and continuous distributions of physical properties (the continuous distribution as the limit case of the discrete with the step going towards zero).

This allow us to allocate the next invariants of physical models development: localised in point, linear, superficial, volumetric distributions of physical properties. At the level of meta-metamodel, each such the concept will represent relevant geometrical model, named the point, the line, the surface and the volume. At the levels of metamodel and model development, these geometrical structures are the part of the expert and user interfaces.

Table 1 shows the examples of physical models, which we can derive from the proposed metamodel. 
Table 1.Analyses of physical models by spatial distribution of properties

\begin{tabular}{|c|c|c|}
\hline $\begin{array}{l}\text { Distribution } \\
\text { of physical } \\
\text { properties }\end{array}$ & $\begin{array}{l}\text { The concept } \\
\text { of meta- } \\
\text { metamodel }\end{array}$ & $\begin{array}{l}\text { The physical abstraction } \\
\text { or model }\end{array}$ \\
\hline 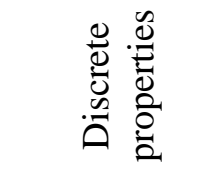 & \multirow[b]{2}{*}{ Point(s) } & $\begin{array}{l}\text { Material point. } \\
\text { Point charge. } \\
\text { Source of oscillations etc. }\end{array}$ \\
\hline \multirow{4}{*}{ 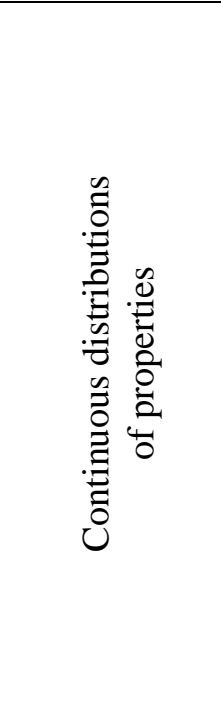 } & & $\begin{array}{l}\text { System of material points. } \\
\text { Dipole, model of a polar and non-polar molecule. } \\
\text { Model of substance (an ideal gas, an ideal liquid, model of } \\
\text { absolutely solid and elastic body). } \\
\text { Electronic gas, model of an electric current etc. }\end{array}$ \\
\hline & Line & $\begin{array}{l}\text { The model of a thread, of a string, of a chain. } \\
\text { Ideal conductor, contour with a current. } \\
\text { Other abstractions - e.g. trajectory, a force line of a field etc. }\end{array}$ \\
\hline & Surface & $\begin{array}{l}\text { Surface distributions of a charge (model of condenser), of a } \\
\text { mass (e.g. for calculations of inertia moment) and other } \\
\text { physical properties. }\end{array}$ \\
\hline & $\begin{array}{l}\text { Volume (3D } \\
\text { region) }\end{array}$ & $\begin{array}{l}\text { Vector (field of force, field of intensity, field of speed) and } \\
\text { scalar fields (field of scalar potential, distribution of intensity } \\
\text { of light wave) etc. }\end{array}$ \\
\hline
\end{tabular}

From the Table 1, follows that operating geometrical abstractions allow us to develop physical models, having different spatial structure. Thus, we can define the method of metamodelling as concretisation of the abstract meta-metamodel concepts by attributing those physical properties (having values at the level of the model development).

Let's note here one important feature of physical modelling. If the development of a model of a material body (e.g. elastic chain) needs specification of functional dependences between separate elements of the system, development of the simulation model of e.g. ideal gas (on the base of laws of collisions of ideal smooth balls), needs implementation of genetic (causal) relationships. In other words, the development of the model of the ideal gas needs the use of the concept of conditional events, which can take place in the modelled system: if coordinates of "molecules" of a gas will match, then their speed is calculated up according to the laws of collision of ideally smooth balls. The metamodelling approach for modelling behaviour of physical systems will be the part of our future research.

\section{FORMAL SEMANTICS OF A GEOMETRICAL META-METAMODEL AND THE METAMODELLING APPROACH}

The given analyses shows us the invariant geometrical structures, we will use as abstractions of the meta-metamodel for development of physical metamodels and further modelling. 
The idea of the proposed approach is the combination of the geometrical information $\left\{\Gamma_{k}\right\}$, defining the spatial structure of a physical domain and the specific for it information $\left\{F_{k}\right\}$, given on the geometry $\left\{\Gamma_{k}\right\}$, where $k=1 \ldots, K, K$ is the number of objects, composing a model of a physical domain.

We define the set of geometrical objects as

$$
\Gamma_{k}^{n}, k=1, \ldots, K, n=0,1,2,3
$$

In each point of (1) the domain-specific information $F(x, y, z, t)$ about properties of the physical domain is given and described in the form of $F_{k}(x, y, z, t)$, i.e. as the constriction (the narrowing) of the function $F$ on the respective object $\Gamma_{k}^{n}$

$$
\left.F\right|_{\Gamma_{k}^{n}}=\left.F_{k}\right|_{\Gamma_{k}^{n}}, k=\overline{1, K}
$$

where $n$ is the parameter, that shows the dimension of the geometrical object $\Gamma_{k}^{n}$ and in the same time defines the symbol of the meta-metamodel:

- if $n=0$, then $\left\{\Gamma_{k}^{n}\right\}$ is a 0 -dimensional object or the point $P$;

- if $n=1$, then $\left\{\Gamma_{k}^{n}\right\}$ is a 1-dimensional object or the line $L$;

- if $n=2$, then $\left\{\Gamma_{k}^{n}\right\}$ is a 2-dimensional object or the surface $S$;

- if $n=3$, then $\left\{\Gamma_{k}^{n}\right\}$ is the region $R$ in the three-dimensional space $R^{3}$.

In the case of $n=1$ and $n=2$ the narrowing $F_{k}$ are the traces of functions $F(x, y, z, t)$ on the corresponding lines $\Gamma_{k}^{1}$ or surfaces $\Gamma_{k}^{2}$.

These formulas are the definition of the alphabet of the metatypes of the geometrical meta-metamodel $\Omega$.

The full definition of the geometrical meta-metamodel is

$$
\Omega=\{\{M T\},\{G\},\{O\}\}
$$

$M T=\{P, L, S, R\}$, where $P$ is the point, $L$ is the line, $S$ is the surface, $R$ is the $3 \mathrm{D}$ region.

$\Omega$ includes the set of operations O: union $A \cup B$, intersection $A \cap B$, complement $A \backslash B$, and supplement $\bar{A}$, where $A, B$ are the instances of $M T$.

$G$ is the system of relationships, are used for specification of the syntax of the metamodel:

- $\quad$ equality $\mathrm{A}=\mathrm{B}$

- $\quad$ separateness $(A \cap B=\emptyset)$

- $\quad$ strict separateness $\left(A \cap B=\emptyset \wedge A^{\prime} \cap B^{\prime}=\varnothing\right)$

- $\quad$ inside $(\mathrm{A} \subseteq \mathrm{B} \vee \mathrm{B} \subseteq \mathrm{A})$

- $\quad$ strict inside $\left.\left((A \subseteq B \vee B \subseteq A) \wedge A^{\prime} \cap B^{\prime}=\varnothing\right)\right)$

- $\quad$ touchiness $\left(\mathrm{A} \cap \mathrm{B}=\varnothing \wedge \mathrm{A}^{\prime} \cap \mathrm{B}^{\prime} \neq \emptyset\right)$

objects:

where $A, B$ are the geometrical objects and $A^{\prime}, B^{\prime}$ are the boundaries of these 
The meta-metamodel $\Omega$ is used for development of the physical metamodel $\Phi$

$$
\Phi=\{\{T\},\{G\},\{C\}\}
$$

$T$ is the types of geometrical objects, produced from $M T$ inside the grammar $G$ with the help of operations $O$.

$G$ is the set of rules (grammar), defining the syntax of the physical model M.

$C$ is the set of methods for calculation of physical properties.

Development of the physical metamodel $\Phi$ inside the meta-metamodel $\Omega$ has the following steps:

- composition of the elements of $M T$ to create the geometrical types $T$ of the metamodel $\Phi$ (by resizing, parallel moving, rotation, distribution in the space, changing other geometric attributes);

- formulation of the rules of the grammar $G$ by putting restrictions on the instances of the types $T$ (e.g. producing the line segment, limitation of the spatial area by planes, etc.);

- attributing $T$, i.e. adding to the geometrical objects the physical properties, measured by the physical values;

- definition the mathematical methods $C$, applicable for the physical model M.

The model $\mathrm{M}$ of a physical domain is

$$
\mathrm{M}=\{\{\Gamma\},\{F\}\}
$$

Where $\Gamma$ is the set of geometrical objects, defining the spatial structure of the physical domain and $F$ is the set of functions, defining the distributions of physical properties on $\Gamma$.

Specified in the points of the geometrical object $\Gamma_{k}^{n}$ the function $F$ depends on mparameters $s_{0}, s_{1}, \ldots, s_{m}: F_{k}\left(s_{0}, s_{1}, \ldots, s_{m}\right)$, which can be time, some operators or functionals from $F$ (e.g. velocity, acceleration, etc.), or some integral characteristics (e.g. surface, volume, total charge, etc.).

If to the spatial variables we add a parameter of time, we come to considering dynamic mathematical model. If the process is stationary, the time parameter is missing.

Formulated approach allows us to automate the development of approximate solutionsforheterogeneous2D and 3D boundary value problems. It was used for:

- reconstruction of 3D surfaces of bodies based on given radar data;

- reconstruction of the internal structure of the earth's crust using data of seismic tomography;

- reconstruction of the internal structure of the $3 \mathrm{D}$ bodies by $2 \mathrm{D}$ computer tomograms;

- design of metamaterials.

In this paper, we will consider software prototypes, which we developed for solution of last two problems. 


\section{IMPLEMENTATION OF SOFTWARE PROTOTYPES}

The proposed metamodelling approach was implemented in visual modeling environments for solving problems of multidimensional physical domains.

At the program level, geometrical objects were implemented by the basic data structures (a separate physical value, linear, surface and space distributions of physical properties are the data arrays of different dimensions). At the same time, these geometrical objects are the concepts of the user interface at the level of metamodel development.

Figures 1 and 2 illustrate the application of the metamodelling approach for solving problems of computer tomography, namely, reconstruction of the three-dimensional structure of the body and the search for illegal items at customs control. In the both cases, the basic concept of the meta-metamodel, used to build a metamodel was the geometrical concept of the surface. Here, the information about the internal structure of the body was obtained from several tomography images, located on the parallel planes. In the first case, the metamodel allows us to solve the problem to find an image in cross-sections of the body that do not coincide with given from the computer tomograph.

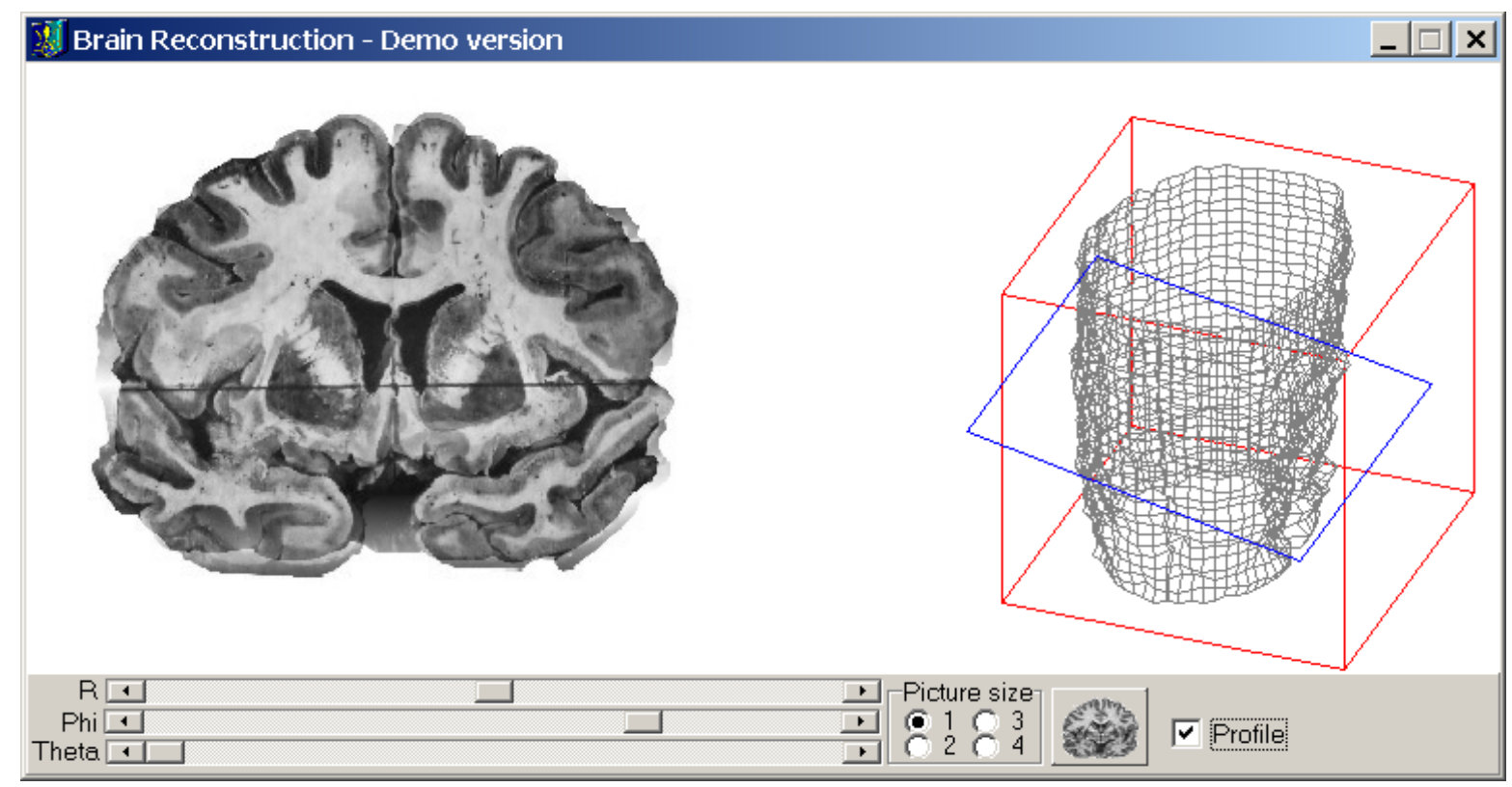

Figure 1. Using the metamodelling approach to solve the problems of computer tomography(reconstruction of the structure of three-dimensional body by the series of its flat cross sections)

In the second case, the geometrical surface was the carrier of physical properties, obtained from the tomograph as two-dimensional image of density distribution, and serves as an input for applying mathematical methods. The details of this approach described in (Patent on invention №78568, 2004).

In general, the process of the model $M$ development inside the metamodel $\Phi$ has the following steps:

- specification of parameters of space and time (number of dimensions, time interval etc.);

- definition of the instances of the types $T$; 
- setting geometrical attributes of the instances of $T$;

- specification of the distributions of $F$ on $\Gamma$ (analytically or as the sets of numerical values);

- generation of the data-structures from the traces of $F$ on $\Gamma$;

- application of the computation algorithms $C$;

- interpretation of the results of modelling and improvement of $\mathrm{M}$.

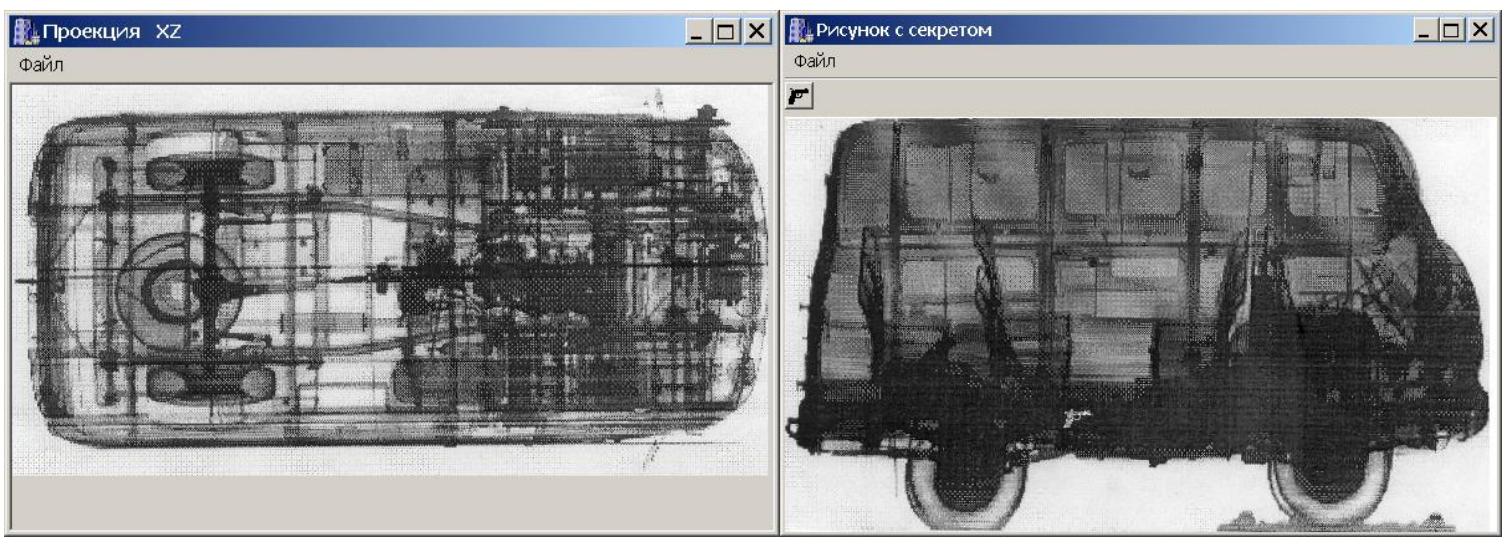

Figure 2. Using the metamodel to solve the problems of computer tomography(search for illegal items at customs control)

Let consider an example of application of metamodelling approach for design of metamaterials (artificial composites with physical properties, which we cannot find in natural materials).

A metamaterial is developed by the inclusion into the natural material (source) of the particles, having any geometrical structure and physical properties. These elements modify the physical properties of the source material, e.g. its permittivity and the magnetic permeability $\varepsilon, \mu$.

Development of the metamodel needs definition of the domain specific-types, applicable for the design of metamaterial. Let's define the types inside the proposed geometrical meta-metamodel (3) as the sets of geometrical objects, having the needed physical properties. Here, domain specific types are the typical inclusions, used for the modelling insertions in the elementary cells of a metamaterial.

Definition of such the types of inclusions is based on existing method of computation (Vitaliy and Felipe, 2010). In our prototype, we use the geometrical objects, whose form-factor can be analytically calculated by

$$
F(\vec{q})=\frac{1}{V_{c}} \int_{V_{\text {inc }}} e^{-i \vec{q} r} d r
$$

For this purpose we define concrete instances of the 3D region (being the base object of the proposed meta-metamodel $\Omega$ ):

-Sphere (with the centre point and the radius as attributes). With the radius $\rightarrow$ 0 we will have transition to the point object. 
-Parallelepiped (with the centre point, the sizes of sides, and the angles of rotation as attributes). With the width $\rightarrow 0$ and the height $\rightarrow 0$ we will have the transition to the line object.

-Cylinder (with the centre point, the length, the radius 1 , the radius 2 , and the angles of rotation as attributes). With the radius $s_{1} \rightarrow 0$ and the radius $s_{2} \rightarrow 0$ we will have transition to the line object.

-Cone (with the centre point, the length, the radius 1 , the radius2, and the angles of rotation as attributes). With the radius $_{1} \rightarrow 0$ and the radius $_{2} \rightarrow 0$ we will have transition to the line object.

The instances of the 3D region of the meta-metamodel (i.e. sphere, parallelepiped, cylinder, and cone) are used for building geometry $\Gamma$ of inclusions in a unit cell. The method for metamaterials modelling as photonic crystals has the following steps:

1. Building geometry $\Gamma$.

2. Setting distributions of the permittivity and the permeability functions $\varepsilon(\vec{r}), \mu(\vec{r})$ among the geometry $\Gamma$. In the simplest case: $\varepsilon\left(\Gamma_{k}\right)=$ const, $\mu\left(\Gamma_{k}\right)=$ const, $k=\overline{1, N}, N-$ the number of objects.

3. Application of the computation algorithm $C$.

Modelling metamaterials as phononic crystals is the same, except we set distributions of the density and sound velocity functions $\rho(\vec{r}), c_{l}(\vec{r})$ and $c_{t}(\vec{r})$ among the geometry $\Gamma$. In the simplest case: $\rho\left(\Gamma_{k}\right)=$ const $, c_{l}\left(\Gamma_{k}\right), c_{t}\left(\Gamma_{k}\right)=$ const $, k=\overline{1, N}, N$-the number of objects

Application of a geometrical metamodel for development of the model of metamaterial as magnetometallic thin wires is shown on Figure 3.By the composition of thin wires the cubic lattice of an isotropic left-handed metamaterial is designed. After visual modelling and setting the distribution of permittivity and permeability we apply the method of calculation of refractive index to explore the specific optical properties of the designed metamaterial (the input to the computation method were the periodic functions of the permittivity and permeability via coordinates).Using other geometrical objects we can design other structures (e.g. with surface, a user can design a super lattice, composed of alternating plates etc.).

Note that, following our approach, the same meta-metamodel can be applied for the different physical problems. For example, the geometrical structure, shown in Figure 3, can be considered as a media of space distribution of an electric charge. Application of Coulomb law and superposition principle allows us to explore properties of the emerged electric field.

Another important feature of the metamodelling approach is the possibility of modelling heterogeneous systems. Interesting example is a design of cyber-physical systems, where geometrical metamodel for physical modelling was combined with a graph based metamodel for software systems design (Vitaliy and Refik, 2013). 


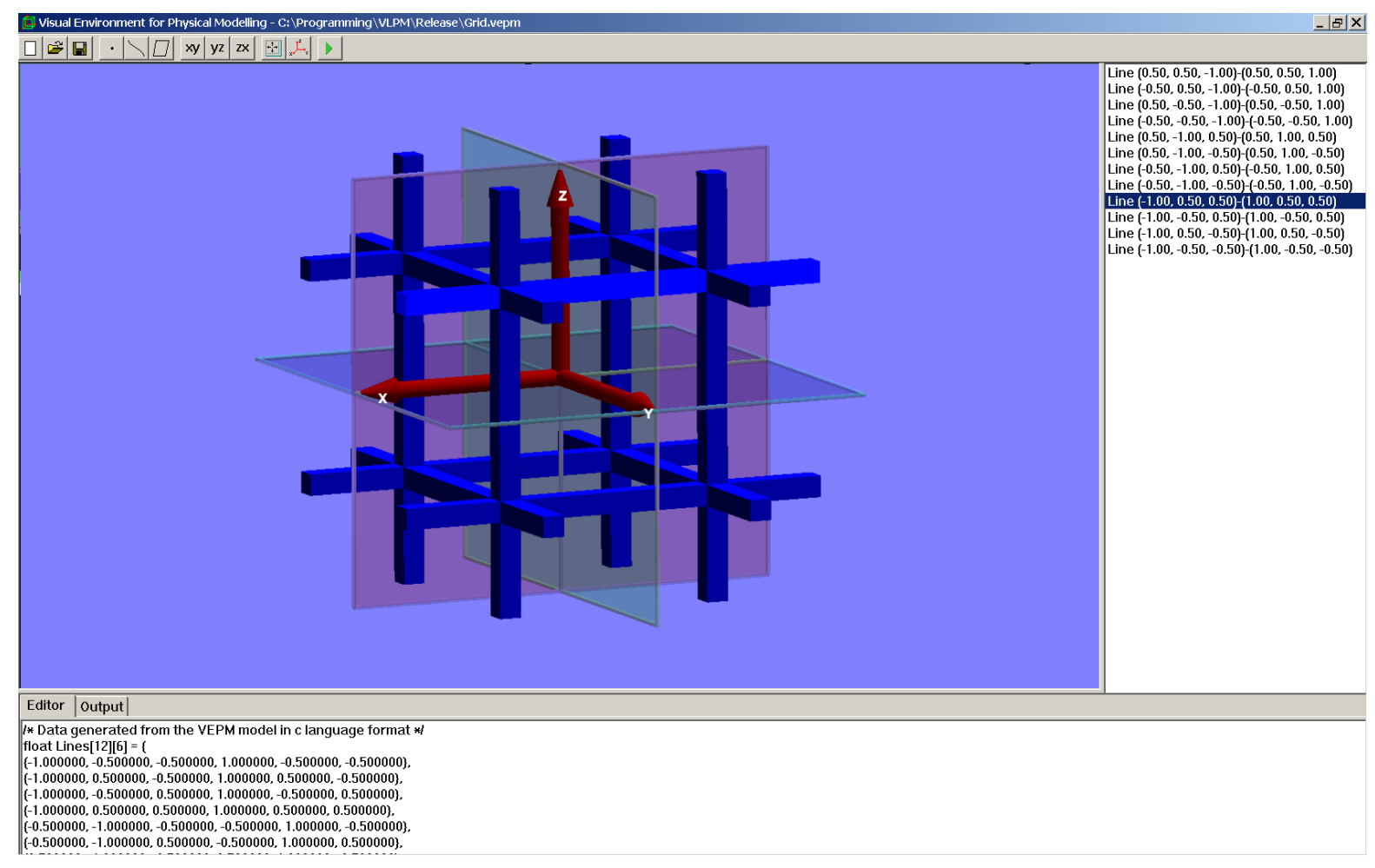

Figure 3. Visual design of metamaterial as a cubic magnetometallicwire lattice

\section{DISCUSSION AND FURTHER TASKS}

Let summarize the advantages of the metamodelling approach:

- Visual rapid development of a physical model.

- Possibility of using by people, having no specific mathematical knowledge.

- Mathematical generality - possibility of application of different mathematical methodsfor geometrical distributions of physical properties.

- Technical generality: possibility of using any programming language and compiler for the implementation of the computation algorithm.

We may briefly summarize the future tasks of development as:

1. Development of an algorithm for calculation of cross sections of geometrical structures (see for example the picture $a$ on the Figure 4). In the current approach, we use geometrical objects, whose form-factor can be analytically calculated. In the case of complex geometrical structures or in the case of cross sections of geometrical objects, this task becomes important.

2. Development of an algorithm for optimal discretisation of cross sections of complex geometrical structures (picture $b$ on the Figure 4). This task means the choice the minimal amount of geometrical objects for discretisation, whose form is maximally, fit the form of approximated geometrical structures.

3. Development of an algorithm for precise allocation of one geometrical object in another (picture c on the Figure 4).

4. Expansion of the set of rules $R$ for models development. Because geometrical structures are invariants with respect to physical models, it gives the possibility of taking into account such the space properties as symmetry, periodicity, chirality etc. 


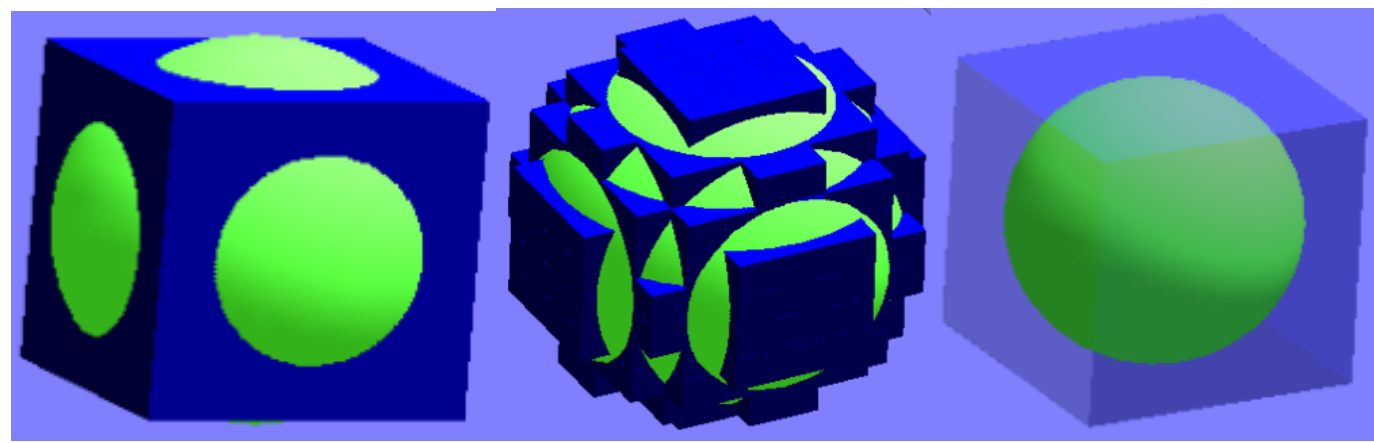

Figure 4. Further tasks for development (pictures a, b, c from left to right)

\section{CONCLUSIONS}

In the paper, we have discussed an applicability of metamodelling approach for development of software tools for physical modelling and simulation. Analyses of physical models allows us to define the geometrical meta-metamodel for development of physical metamodels and physical modelling. The mathematical base of the proposed approach is a combination of geometrical information about the spatial structure of physical objects $\left\{\Gamma_{k}\right\}$ and domain-specific information $\left\{F_{k}\right\}$, given on these geometrical objects. The advantage of the proposed metamodelling approach is the development of computer tools, which accelerate the process of physical research through the visual design and use of different mathematical methods. The applicability of the metamodelling approach for solving task of computer tomography and design of metamaterials is shown.

\section{REFERENCES}

UML (www.uml-forum.com)

MatLab (www.mathworks.com)

MathCAD (www.mathsoft.com)

Maple (www.maplesoft.com)

Catalog of UML Profile Specifications.

http://www.omg.org/technology/documents/profile_catalog.htm

Steven Kelly and Juha-Pekka Tolvanen.(2008). Domain-Specific Modeling: Enabling Full Code Generation. Wiley-IEEE Computer Society Pr. 427 p.

Gregory G. Nordstrom.(1999). Metamodeling - Rapid Design and Evolution of DomainSpecific Modeling Environments. Dissertation for the Degree of Doctor of Philosophy i

n Electrical Engineering. Nashville, Tennessee- 170 p.

Vitaliy Mezhuyev, RefikSamet.(2013).Geometrical Meta-metamodel for Cyber-Physical Modelling. Proceedings of International Conference Cyberworlds 2013 (Yokohama, Japan, October 21-23, 2013). pp. 89-93.

Vitaliy Mezhuyev.(2014).Methodology of Domain Specific Mathematical Modelling. Proceedings of the 3rd International Congress on Natural Sciences and Engineering ICNSE 2014 (Kyoto, Japan, May 7-9, 2014). Pp. 54-64.

Vitaliy Mezhuyev.(2015)Metamodelling Architecture for Modelling Domains with Different Mathematical Structure. Advanced Computer and Communication 
Engineering Technology. Lecture Notes in Electrical Engineering. Vol. 315. Pp. 1049-1055.

Vitaliy Mezhuyev.(2014) Architecture of Software Tools for Domain-Specific Mathematical Modelling. Proceedings of 2014 International Conference on Computer, Communication, and Control Technology (Langkawi, Malaysia, Sept 24, 2014). Pp. 166-170.

Patent on invention No.78568, Ukraine. (2007). The Method to Restore the Internal Structure of a 3D Object. Ivan Sergienko, Oleg Lytvyn, Vitaliy Mezhuyev. No.20041210438. - Decl. 20.12.2004. - Publ. 10.04. - Issue. № 4.

Vitaliy Mezhuyev, Felipe Pérez-Rodríguez.(2010). Visual Environment for Metamaterials Modelling. Some current topics in condensed matter physics. Universidad Autónomadel Estado de Morelos. - 1-13 pp. 